\title{
Efeito de diferentes diluidores sobre a membrana plasmática do espermatozóide eqüino e fertilidade do sêmen resfriado
}

\section{Effect of different extenders on sperm plasma membrane and fertility of equine cooled semen}

\author{
Monique de Albuquerque Lagares, ${ }^{*}$ Luciana Silva Meirelles, ${ }^{\star *}$ Vera Beatriz Wald, ${ }^{\star *}$ Ricardo Macedo Gregory, ${ }^{\star *}$ \\ Rodrigo Costa Mattos**
}

\begin{abstract}
Resumo
O sêmen de garanhões mostra diferenças individuais com relação a sua preservação. Apesar das causas ainda não estarem claras, fenômenos que ocorrem durante manipulação e armazenagem do sêmen $a+5^{\circ} \mathrm{C}$ exercem considerável influência sobre a funcionalidade e integridade da membrana plasmática do espermatozóide eqüino. O presente trabalho objetivou avaliar a eficácia da adição de diferentes diluentes de sêmen sobre a motilidade progressiva, manutenção da funcionalidade e integridade da membrana plasmática do espermatozóide eqüino preservado $a+5^{\circ} \mathrm{C}$ por 72 horas, bem como sobre a taxa de fertilidade. Pode-se concluir que a avaliação da integridade e funcionalidade da membrana espermática oferece informações úteis à análise de rotina do sêmen eqüino que podem auxiliar na previsão da fertilidade do sêmen resfriado.
\end{abstract}

Palavras-chave: teste hiposmótico; sêmen eqüino; membrana plasmática; diluidor; fertilidade.

\begin{abstract}
Stallions semen shows individual differences regarding to its preservation suitability. Although the causes for that are not clear, phenomena that occur during semen manipulation and storage in $+5^{\circ} \mathrm{C}$ exerce considerable influence on functional and morphological integrity of equine sperm plasma membrane. Besides fertility rate, the present work evaluated the efficiency of different semen extenders on spermatozoal motility, plasma membrane functional and morphological integrity of equine liquid preserved semen at $+5^{\circ} \mathrm{C}$ for $72 \mathrm{~h}$. It could be concluded that sperm functional and morphological integrity evaluation is usefull for equine semen rotine analysis and could aid to preview the fertility suitability of cooled semen.
\end{abstract}

Keywords: hyposmotic swelling test; equine semen; plasma membrane; extender, fertility.

\section{Introdução}

Os parâmetros convencionais de análise de sêmen como concentração, motilidade, morfologia e vitalidade espermática possuem limitada capacidade de previsão da fertilidade masculina (Blasco, 1984; Van der Ven et al., 1986; Amann 1989). Alterações na composição e na integridade da membrana espermática ocorrem durante o processo de fertilização, manipulação e armazenagem de sêmen. Sabendo-se que a membrana plasmática está envolvida com trocas metabólicas com o meio, o seu estudo é de grande importância para se aprimorar os índices de fertilidade. Vários autores estudaram a funcionalidade da membrana plasmática do espermatozóide eqüino (Zavos, 1991; Noiles et al, 1992; Lagares et al, 1994; Lagares, 1995; Kohne et al., 1995; Watson, 1996; Caiza de la Cueva et al., 1997), mas poucos avaliaram se a sua integridade era influenciada com a adição de diferentes diluentes de sêmen (Lagares et al, 1999).
O presente trabalho objetivou avaliar a eficácia da adição de diferentes diluentes de sêmen na manutenção da motilidade progressiva, integridade e funcionalidade da membrana plasmática do espermatozóide eqüino preservado $\mathrm{a}+5^{\circ} \mathrm{C}$ por 72 horas, bem como a fertilidade do sêmen resfriado por 24 horas.

\section{Material e métodos}

A avaliação in vitro do presente experimento foi desenvolvida nas dependências da Faculdade de Veterinária da Universidade Federal do Rio Grande do Sul e Posto de Fomento Agrovin, Porto Alegre, RS, e foi conduzida de junho de 1997 a maio de 1998. O teste in vivo foi realizado em um haras de cavalos da raça árabe situado a 90 quilômetros de Porto Alegre, no período de novembro de 1997 a fevereiro de 1998.

Foram coletados 50 ejaculados de cinco garanhões. Após cada coleta, o sêmen foi imediatamente avaliado quanto à motilidade, vigor, volume e concentração espermática. Cada

\footnotetext{
* Escola de Veterinária da UFMG- Depto. de Clínica e Cirurgia Veterinária-Setor de Reprodução Animal Av. Antônio Carlos, 6627- CEP 31270-901Belo Horizonte, MG, e-mail:mlagares@ vet.ufmg.br

** Faculdade de Veterinária da UFRGS - Laboratório de Reprodução Animal. Av. Bento Gonçalves, 9090, CEP 91540-000, Porto Alegre, RS, e-mail: rcmattos@vortex.ufrgs.br
} 
ejaculado foi dividido em quatro amostras de igual volume, que sofreram diluição mínima de 3:1 de maneira a se alcançar uma concentração final de 25 a $50 \times 10^{6}$ espermatozóides/ $\mathrm{ml}$, com cada um dos seguintes diluentes:

- Leite: leite em pó desnatado, reconstituído com água bidestilada, deionizada a 10\% (251 mOsmol/l) (Mattos, 1995);

- Tyrode: $65 \%$ de leite (Kenney et al.,1975) e 35\% de Tyrode modificado (Padilla e Foote, 1991) adicionado com $2 \%$ de gema de ovo (Gather, 1994) (402 mOsmol/l);

\section{- Glicina (Van Der Holst, 1984) (290 mOsmol/l) e}

- Kenney (Kenney et al.,1975) (327 mOsmol/l).

Nenhum dos diluentes recebeu a adição de antibióticos. Foi realizada a medição do $\mathrm{pH}$, que foi ajustado, quando necessário, para um valor entre 6,7 e 7,2. O sêmen foi avaliado quanto a motilidade espermática progressiva antes e após a adição dos diluentes (Oh) e as 24,48 e 72 horas posteriores à diluição. Concomitante a cada avaliação de motilidade, uma alíquota foi separada para se verificar a integridade da membrana plasmática através da coloração diacetato 6 carboxifluoresceína (6-CFDA) (Kneissl,1993) com auxílio de microscópio de fiuorescência e funcionalidade da membrana plasmática através do teste hiposmótico com água destilada (Lomeo e Giambersio, 1991) modificado por Lagares et al. (1998) na diluição 1:3 (sêmen:água destilada). O resfriamento das amostras $\mathrm{a}+5^{\circ} \mathrm{C}$ foi realizado após a primeira avaliação do sêmen, perfazendo uma curva de $1^{\circ} \mathrm{C} /$ $\min$.

Devido à intensa utilização dos meios Leite e Kenney na inseminação artificial com sêmen resfriado, além de apresentarem os melhores resultados, a maioria dos testes realizados in vitro, optou-se por testá-los in vivo.

A taxa de fertilidade foi avaliada por meio da inseminação artificial de três grupos de éguas: Grupo 1: inseminado com sêmen fresco diluído no dia da coleta (Oh) e Grupos $\underline{2}$ e $\underline{3}$ : inseminados com sêmen adicionado dos diluentes Leite $e$ Kenney, respectivamente, descritos anteriormente, preservados a $+5^{\circ} \mathrm{C}$ durante $24 \mathrm{~h}$.

Para tal, foram utilizadas 69 éguas reprodutivamente sadias, com idades variando entre três a 20 anos e três garanhões aptos à reprodução ( 6 a 14 anos de idade). A dose inseminante mínima utilizada foi de $500 \times 10^{6}$ espermatozóides viáveis. A primeira inseminação foi realizada nas éguas em cio no dia em que foi detectado um folículo maior ou igual a 35 $\mathrm{mm}$ de diâmetro e as inseminações foram repetidas a cada 48 horas até a detecção da ovulação. O diagnóstico de gestação foi realizado por palpação retal e ultra-sonografia.

\section{Análise estatística}

Para comparar a motilidade progressiva, vigor e a porcentagem de espermatozóides com membrana funcional e íntegra entre os tratamentos nos diferentes tempos, foi utilizada a metodologia de Modelos Mistos, sendo que as médias foram comparadas pelo procedimento LSMEANS do programa SAS. $A$ taxa de prenhez obtida nos dois tempos de preservação ( 0 e 24 horas) foi comparada pelo teste de qui-quadrado. As diferenças foram consideradas significativas no nível de probabilidade 0,05 .

\section{Resultados e discussão}

Embora não tenha ocorrido interação entre os graus de diluição e os diluentes utilizados, foi observada interação do tempo de armazenamento do sêmen com os diluentes e com os graus de diluição. Os diluentes influenciaram de maneira diferente a integridade e funcionalidade da membrana plasmática do espermatozóide observadas pela coloração de 6-carboxifluoresceína-diacetato (6-CFDA) e pelo teste hiposmótico, respectivamente. Embora alguns autores (Pickett e Amann, 1987) afirmem que o diluente de sêmen adequado deva possuir osmolaridade entre 277-326 $\mathrm{mOsmol} / \mathrm{l}$, não foi observada diferença significativa quanto a porcentagem de espermatozóides com membrana plasmática funcional (Tabela 1) entre o diluente que apresentou a osmolaridade mais baixa, Leite $(251 \mathrm{mOmol} / \mathrm{l})$ e a mais alta, Tyrode (402 $\mathrm{mOsmol} / \mathrm{l})$, demonstrando uma provável maior influência dos componentes dos diluidores sobre a manutenção da funcionalidade da membrana, do que da osmolaridade do mesmo (Jeyendran et al., 1984). Quando a preservação do sêmen fresco no diluente de Kenney foi comparada com a do plasma seminal, não se observou diferença significativa quanto a vitalidade do espermatozóide eqüino (Lagares et al., 1998). No presente trabalho, o diluente Kenney apresentou a menor taxa de espermatozóides que reagiu positivamente à condições hiposmóticas, mostrando menor capacidade de manutenção da funcionalidade da membrana plasmática quando comparado aos outros diluentes (Tabela 1). No entanto, parece não ter sido o suficiente para afetar significativamente a taxa de prenhez entre os três grupos de éguas inseminadas com sêmen fresco Oh; sêmen resfriado com Leite 24 horas e sêmen resfriado com Kenney 24 horas (Tabela 4). Por outro lado, o resultado da prenhez pode ter sido mascarado pelo alto número de espermatozóides viáveis/inseminação (>500 $\times 10^{6}$ espermatozóides/dose) e, portanto, acima dos requerimentos mínimos (100 × $10^{6}$ espermatozóides/dose).

Por meio da coloração 6-CFDA foi possível detectar uma influência diferente de cada diluidor sobre a integridade da membrana plasmática até mesmo antes do resfriamento (Oh) (Tabela 2). Os diluentes de Leite e de Kenney não se diferenciaram significativamente quanto a manutenção da integridade da membrana, o que pode explicar a taxa de prenhez semelhante entre os grupos 2 e 3 de éguas (Tabela 4). Por outro lado, o reduzido percentual de espermatozóides com membrana plasmática íntegra, observado após a adição do diluente Glicina e durante as 72 horas de preservação a $+5^{\circ} \mathrm{C}$, pode explicar a significativa baixa taxa de prenhez obtida no trabalho anterior (Keller, 1998). Neste, foi comparada a taxa de prenhez com inseminação artificial do grupo de éguas com sêmen preservado com diluente Leite e Glicina $\mathrm{a}+5^{\circ} \mathrm{C}$ por 24 horas, respectivamente. Vale a pena enfatizar que a motilidade espermática observada nos dois diluentes eram semelhantes, porém a taxa de prenhez com diluente de Glicina $(55,5 \%)$ foi significativamente $(p<0,05)$ 
inferior à obtida com a inseminação com sêmen adicionado do diluente Leite (83\%).

A partir de 24 horas de preservação, foi observada motilidade progressiva (Tabela 3 ) estatisticamente superiores no sêmen adicionado dos diluentes de Leite e Kenney quando comparado aos de Tyrode e de Glicina, mostrando que os dois primeiros diluidores oferecem uma melhor proteção aos espermatozóides a reduções bruscas de temperatura (curva rápida de resfriamento). É sabido que a proporção de eletrólitos e não-eletrólitos do meio influencia a motilidade e a fertilidade (Pickett et al., 1975). De acordo com os resultados do presente experimento, esta proporção deve estar mais adequada nos diluentes de Leite e de Kenney.

Tabela 1: Porcentagem de espermatozóides com membrana funcional observada com o teste hiposmótico na diluição de 1:3 em diferentes diluentes e tempos até 72 horas

\begin{tabular}{|c|c|c|c|c|}
\hline \multirow[t]{2}{*}{ Diluentes } & \multicolumn{4}{|c|}{ Tempo (h) } \\
\hline & 0 & 24 & 48 & 72 \\
\hline Leite & $57,2 \pm 14,8^{a}$ & $52,5 \pm 12,7^{a}$ & $45,4 \pm 13,4^{a}$ & $36,5 \pm 14,6^{a}$ \\
\hline Kenney & $43,3 \pm 18,2^{b}$ & $33,8 \pm 15,2^{b}$ & $25,0 \pm 13,5^{b}$ & $17,3 \pm 8,4^{b}$ \\
\hline Tyrode & $56,1 \pm 11,8^{\mathrm{a}}$ & $48,1 \pm 14,0^{a}$ & $36,7 \pm 16,4^{c}$ & $27,2 \pm 15,7^{\circ}$ \\
\hline Glicina & $66,9 \pm 11,1^{c}$ & $53,1 \pm 13,5^{a}$ & $36,3 \pm 16,2^{c}$ & $25,2 \pm 14,3^{c}$ \\
\hline
\end{tabular}

abc letras diferentes na mesma coluna se diferenciam significativamente $(p<0,05)$

Tabela 2: Porcentagem de espermatozóides com membrana íntegra observada com a coloração de 6-carboxifluoresceínadiacetato em diferentes diluentes e tempos até 72 horas

\begin{tabular}{|ccccc|}
\hline Diluentes & \multicolumn{4}{c}{ Tempo $(\mathrm{h})$} \\
\cline { 2 - 5 } & 0 & 24 & 48 & 72 \\
\hline Leite & $70,9 \pm 14,8^{\mathrm{a}}$ & $62,8 \pm 16,4^{\mathrm{a}}$ & $56,9 \pm 18,9^{\mathrm{a}}$ & $49,5 \pm 19,9^{\mathrm{a}}$ \\
Kenney & $62,8 \pm 20,2^{\mathrm{ab}}$ & $55,5 \pm 22,1^{\mathrm{ab}}$ & $47,1 \pm 25,5^{\mathrm{ab}}$ & $35,5 \pm 26,3^{\mathrm{ab}}$ \\
Tyrode & $54,4 \pm 15,7^{\mathrm{b}}$ & $45,2 \pm 15,3^{\mathrm{b}}$ & $40,2 \pm 15,5^{\mathrm{b}}$ & $34,2 \pm 16,2^{\mathrm{b}}$ \\
Glicina & $30,4 \pm 15,7^{\mathrm{c}}$ & $24,4 \pm 13,8^{\mathrm{c}}$ & $20,112,7^{\mathrm{c}}$ & $17,4 \pm 14,1^{\mathrm{c}}$
\end{tabular}

ab letras diferentes na mesma coluna se diferenciam significativamente $(p<0,05)$

Agradecimentos à FAPERGS pelo apoio financeiro.

\section{Referências bibliográficas}

AMANN R.P. Can the fertility potencial of a seminal sample be predicted accurately? J. Androl., v. 10, p. 89-98, 1989.

BLASCO L. Clinical tests of sperm fertilizing ability. Fertil Steril, v. 41, p. 177-192, 1984.
Tabela 3: Porcentagem de espermatozóides com motilidade progressiva em diferentes diluentes e tempos até 72 horas

\begin{tabular}{|c|c|c|c|c|}
\hline \multirow[t]{2}{*}{ Diluentes } & \multicolumn{4}{|c|}{ Tempo (h) } \\
\hline & 0 & 24 & 48 & 72 \\
\hline Leite & $50,6 \pm 11,6^{a}$ & $29,5 \pm 10,8^{a b}$ & $19,7 \pm 9,8^{a}$ & $11,6 \pm 9,0^{a}$ \\
\hline Kenney & $51,4 \pm 11,6^{a}$ & $31,9 \pm 11,8^{a}$ & $21,5 \pm 11,7^{a}$ & $11,8 \pm 9,6^{a}$ \\
\hline Tyrode & $45,4 \pm 10,5^{a}$ & $13,5 \pm 9,7^{c}$ & $3,6 \pm 4,6^{b}$ & $1,1 \pm 2,7^{b}$ \\
\hline Glicina & $51,5 \pm 11,5^{a}$ & $25,9 \pm 13,1^{b}$ & $13,5 \pm 10,5^{c}$ & $5,2 \pm 6,6^{b}$ \\
\hline
\end{tabular}

abc letras diferentes na mesma coluna se diferenciam significativamente $(p<0,05)$

Tabela 4: Percentual de éguas prenhes durante estação de monta 1997-1998 inseminadas com os diluentes Leite Oh, Leite 24 horas e Kenney 24 horas

\begin{tabular}{|lccc|}
\hline Diluentes & \% éguas prenhes & $\begin{array}{c}\mathrm{n}^{\circ} \text { de éguas } \\
\text { prenhes }\end{array}$ & $\begin{array}{c}\mathrm{n}^{\circ} \text { de éguas } \\
\text { inseminadas }\end{array}$ \\
\hline Leite $0 \mathrm{~h}$ & $79,16^{\mathrm{a}}$ & 19 & $(24)$ \\
Leite $24 \mathrm{~h}$ & $62,50^{\mathrm{a}}$ & 15 & $(24)$ \\
Kenney 24h & $57,14^{\mathrm{a}}$ & 12 & $(21)$
\end{tabular}

${ }^{a}$ letras iguais na mesma coluna não se diferenciam significativamente $(p>0,05)$

\section{Conclusões}

O presente trabalho mostrou que o diluente Leite ofereceu os melhores resultados tanto da preservação da motilidade progressiva, quanto da funcionalidade e integridade da membrana plasmática do espermatozóide durante as 72 horas de armazenamento $\mathrm{a}+5^{\circ} \mathrm{C}$, bem como taxa de prenhez satisfatória, quando comparado aos outros diluentes avaliados. Além disso, o teste hiposmótico e a coloração de 6CFDA parecem avaliar aspectos diferentes da membrana plasmática. Como a redução da temperatura durante o processo de resfriamento altera a composição e estrutura da membrana plasmática do espermatozóide, a utilização conjunta do teste hiposmótico e de colorações como a 6-CFDA associada aos parâmentros convencionais de análise do sêmen é indicada como forma de se obter maiores informações sobre a preservação do sêmen resfriado eqüino e, conseqüentemente, aperfeiçoar a inseminação artificial, para que futuramente resultados semelhantes com a utilização do sêmen congelado possam ser obtidos.
CAIZA DE LA CUEVA, F.I., RIGAU, T., BONET, S., MIRÓ, J.,BRIZ, M., RODRÍGUEZ-GIL, J.E.Subjecting horse spermatozoa to hypoosmotic incubation: effects of ouabain. Theriogenology, v. 47, p. 765-784, 1997.

GATHER, C. Einfluß eines mittels Tyrodemedium modifizierten Magermilchverdünners auf Motilität, Morphologie und Vitalität 
flüssigkonservierten Hengstspermas. 1994. 131 f.Tese (Doutorado) Tierärztl. Hochschule Hannover.

KELLER, A. Efeito de dois métodos de remoção do plasma seminal, diluentes, e tempo de armazenamento sobre a motilidade e fertilidade espermática. 1998. 120 f. Dissertação (Mestrado) - Faculdade de Veterinária - Universidade Federal do Rio Grande do Sul.

KENNEY, R.M., BERGMANN, R.V., COOPER, W.L., MORSE, G.W. Minimal contamination technique for breeding mares: technique and preliminary findings. Proceedings, Am Assoc Equine Pract., v. 21, p. 327-336, 1975.

KOHNE, K.,PETZOLDTR., SIEMEH., TÖPFER-PETERSENE., KLUGE. Osmotische Resistenz von Hengstspermien und deren Beziehungen zur Flüssig- und Tiefgefrierkonservierungsfähigkeit. Reprod in Domestic Anim., Suppl. 3, p. 128 abstr. 1995.

LAGARESM.A., PETZOLDTR., SIEMEH., KLUGE. Zurhypoosmotischen Belastbarkeit von Spermien des Hengstes. Reprod in Domestic Anim, v. 29, n. 2/3, p. 204, 1994.

LAGARES M.A. Bestimmung der osmotischen Resistenz von Hengstsamenzellen. 1995. 123 f. Tese (Doutorado) Tierärztl Hochschule Hannover.

LAGARES M.A., PETZOLDT R., SIEME H., KLUG E. Preservação do sêmen fresco eqüino: Avaliação da integridade da membrana espermática sob condições hiposmóticas. Arquivos da Faculdade de Veterinária da UFRGS, Porto Alegre, v. 26, n. 1, p. 29-42, 1998.

LAGARES M.A., MEIRELLES, L.S., WALD, V.B., GREGORY, R.M. MATTOS, R.C. Manutenção da motilidade e funcionalidade da membrana plasmática do espermatozóide eqüino no sêmen resfriado com diferentes diluentes. Revista Bras. Reprod.Anim. v. 23, n. 2/3, p.295-297, 1999.

LOMEO A.M., GIAMBERSIO A.M. 'Water-test': a simple method to assess sperm-membrane integrity. Int J Androl, v.14, p. 278-282, 1991.
MATTOS, R. Influência de diferentes métodos de preservação de sêmen eqüino sobre a fertilidade, motilidade espermática e contaminação bacteriana. 1995. 121 f. Dissertação (Mestrado) - Faculdade de Veterinária - Universidade Federal do Rio Grande do Sul.

NOILES, E.E., MAZUR, P., BENKER, F. W., KLEINHANS, F.W., AMANN, R.P.,CRTSER, J.K. Critical osmolality, water, and glycerol permeability coeficient determination of equine spermatozoa. Biol. Reprod., v. 46, p. 95, abstr. 177, 1992.

PADILLA, A. W., FOOTE, R. H. Extender and centrifugation effects on the motility patterns of slow-cooled stallion spermatozoa. J. Anim Sci., v. 69, p. 3308-3313, 1991.

PICKETT, B.W., AMANN, R.P. Extension and storage of stallion spermatozoa: a review. J.Eq Vet Sci., Wildomar - California, v. 7, n. 5, p. 289-302, 1987.

PICKETT, B.W., BURWASH, L.D., VOSS, J.L., BACK, D.G. Effect of seminal extenders on equine fertility. J.Anim Sci., v. 40, n. 6, p. 1136-1146, 1975.

VAN DER HOLST, W. Stallion semen production in A. I. programs in the Netherlands. In: COUROT. M. (ed.): The male in farm animal reproduction. Martinus Nijhoff Publishers, 1984, p.195-201.

VANDER VENH.H., JEYENDRANR.S., AL-HASANIS., PEREZ-PELAEZ M., DIETRICH K., ZANEVELD L.J.D. Correlation between human sperm swelling in hyposmotic medium (hyposmotic swelling test) and in vitro fertilization. J. Androl., v. 7, p.190-196, 1986.

WATSON, P.F. Cryopreservation of spermatozoa. In: Symposium on Stallion Semen Production, Evaluation, and Preservation, Amersfoort, p.19 abstr, 1996.

ZAVOS, P.M. Hypoosmotic swelling test (HOST)/ functional integrity of sperm membrane. Assis. Reprod Technol Androl., v. 2, p. 215-216, 1991. 